Die nationalpolitische Bedeutung der Germanistik im 19. Jahrhundert: Ersetzt statt erforscht - Thesen zu Lehndeutsch, Purismus und Sprachgermanistik

\title{
0. Vorbemerkung
}

Die Thesen in der hier abgedruckten Form (2.) lagen den Teilnehmern der Tagung schriftlich vor, wie auch die tabellarischen Übersichten über Wortentlehnungen und Lehnwortbildungen (3.), und wurden in stark gekürzter Fassung vorgetragen. Nachgetragen wurden die Anmerkungen und weiterführende Literaturhinweise, die die bei thesenartiger Darstellung nicht möglichen Begründungs- und Argumentationszusammenhänge wenigstens andeuten sollen. Die Standortbestimmung (1.) wurde unmittelbar nach der Tagung niedergeschrieben, um den frischen Eindruck der Diskussion und mir wesentlich Erscheinendes festzuhalten. Sie ist nicht etwa als solipsistische Bekenntnis zu verstehen, sondern als persönlicher Kommentar zu den apodiktisch formulierten Thesen, der vielleicht zur Klärung des Erkenntnisinteresses bei einem Thema beitragen könnte, das m.E. nur scheinbar sachlich-neutral abgehandelt werden kann.

\section{Zur Standortbestimmung}

Als die Organisatoren das Tagungsprogramm zusammenstellten und das Thema des Podiumsgesprächs festlegten, konnten sie nicht voraussehen, wie aktuell und brisant das Thema bis zur Tagung werden sollte. Betroffen durch die Ereignisse in Europa seit Ende 1989 waren einmal die deutschsprachigen Germanisten aus Österreich, der Schweiz und der Bundesrepublik, vor allem aber aus der DDR, ist man doch durch Ereignisse in dem eigenen Heimatland auf eine ganz besondere Art und Weise betroffen. So standen ja zur Zeit der Tagung die Wissenschaftler aus der DDR unmittelbar vor entscheidenden, freien und geheimen Wahlen. Betroffen waren aber auch die Germanisten aus dem Ausland, allen voran wohl diejenigen aus den angrenzenden (kleineren) Ländern wie Dänemark und den Niederlanden, Polen und der Tschechoslowakei. Auffällig war jedoch, daß während der Podiumsdiskussion praktisch nur Deutsche sich zu Wort meldeten. Die Diskussion beeindruckte durch Offenheit und Ehrlichkeit, durch das Bemühen um Sachlichkeit und zurückhaltende Formulierung. Wie nicht anders zu erwarten - und von den Organisatoren auch so vorgesehen - war, blieb die Diskussion nicht beim 19. Jahrhundert stehen, sondern bezog das 20. Jahrhundert mit ein. Die 
Frage nach einem bzw. dem deutschen "Sonderweg" wurde mehrfach gestellt. Beantwortet wurde sie trotz verschiedener Ansätze nicht, was nicht verwunderlich ist, denn dieser „Sonderweg" ist m.E. letztlich nicht rational begründbar oder erklärbar. Auch wir Germanisten, die wir uns mit der Vergangenheit unseres Fachs auseinandersetzen, sind meiner Überzeugung nach hierdurch überfragt. Nichtsdestotrotz scheint es mir notwendig, daß diese Auseinandersetzung ständig fortgesetzt und die Frage nach der nationalpolitischen Bedeutung der Germanistik immer wieder auf die Tagesordnung gesetzt wird.

Bei dieser Auseinandersetzung sollten wir nicht nur eher historiographisch die Vergangenheit aufarbeiten, indem wir z.B. die Handlungen früherer Germanistengenerationen unter die Lupe nehmen, deren Beweggründe aufspüren und deren Folgen aufzeigen, sondern wir sollten vor allem auch sub specie historiae unser eigenes germanistisches Tun und Lassen in der Gegenwart reflektieren. Denn dieses können wir notfalls noch revidieren und ändern, jenes dagegen nicht mehr. Dementsprechend geht es mir primär um die Lehren, die die Geschichte der Gegenwart aufgibt. Dies gilt ganz allgemein, erst recht jedoch für das Thema des Podiumsgesprächs: „Die nationalpolitische Bedeutung der Germanistik". So hatten es offenbar auch die Organisatoren der Tagung verstanden, denn das Podiumsgespräch war ja ein Beitrag neben anderen zum Tagungsthema: „Das 19. Jahrhundert. Sprachgeschichtliche Wurzeln des heutigen Deutsch".

Mein Beitrag zum Podiums- und Tagungsthema handelt(e) von den fremdsprachlichen lexikalischen Einflüssen auf das Deutsche (im 19. Jahrhundert) und von der Einstellung deutscher Sprachteilhaber diesen Einflüssen gegenüber. Dies war zum einen biographisch bedingt: Sprachlich gesehen war Deutsch für mich neben Latein und Französisch die dritte Fremdsprache, die ich auf Gymnasium und Universität studierte. Sprachwissenschaftlich betrachtet beschäftigte ich mich zuerst vor allem mit Französisch und Englisch. Ich war also schon voreingenommen, als ich zu Deutsch und zur Sprachgermanistik kam. So war ich beispielsweise mit dem lexikalischen Erbe der gräkolateinischen Antike in den modernen europäischen Kultursprachen etwa als hard words bzw. inkhorn terms im Englischen oder als mots savants im Französischen vertraut. Das sind Bezeichnungen, die in erster Linie Sprachsoziologisches, die Geltung dieses Erbes im Spracherwerb und Sprachgebrauch, zum Ausdruck bringen. Im Deutschen dagegen gelten sie als Fremdwörter. Der deutsche Begriff des Fremdworts, der primär auf dem Merkmal Herkunft beruht und Sprachsoziologisches somit hinter Etymologisches zurücktreten läßt, war mir u.a. deshalb fremd; es war mir eben ein Fremd- 
wort. Grund genug, mich wissenschaftlich damit auseinanderzusetzen in dem Versuch, eine - vom Latein, Französischen und Englischen her beeinGußte - Außenperspektive in die germanistische Diskussion einzubringen: Aufgrund anderer Sprach- und Fremdsprachenkenntnisse sowie eventuell auch anderer linguistischer Kompetenz ist der Auslandsgermanist in einer anderen Lage als sein deutscher oder deutschsprachiger Kollege; er benutzt, betrachtet, beurteilt und bewertet das Deutsche zwangsläufig anders als der native speaker.

Die Wahl des Themas hatte zum zweiten mit der Bringschuld der Wissenschaft(ler) zu tun. Die sog. Fremdwortfrage beschäftigt nämlich deutsche Sprachteilhaber offensichtlich sehr, wie man an der Häufigkeit der Zeitungsglossen und -briefe über, meist gegen das Fremdwort oder aber an der Vielzahl und Vielfalt der heutigen Fremdwörterbücher ablesen kann: So erschien z.B. rechtzeitig zur Tagung wieder eine neubearbeitete und erweiterte Auflage des Duden-Fremdwörterbuchs, die fünfte bereits seit 1960. Mit dem Interesse der breiten Öffentlichkeit kann also gerechnet werden. Die Fremdwortfrage ist aktuell, was im 19. Jahrhundert auch nicht anders war, und fordert sprachkritische Germanisten aus dem Elfenbeinturm fachinterner Wissenschaftsdebatten heraus. Dies gilt umsomehr, als es den Germanisten m.E. doch gelingen müßte, den Nachweis dafür zu erbringen, daß einerseits in der Diachronie die Fixierung auf das Fremdwort den Blick für das eigentümliche Zusammenspiel von Entlehntem und Ererbtem in der Herkunftsstruktur des deutschen Wortschatzes versperrt, indem sie u.a. Inhaltsseitiges bzw. das innere Lehngut außer Acht läßt; und daß andererseits in der Synchronie die Fixierung auf das Fremdwort von dem brisanten Problem der explodierenden Fachwortschätze und der zunehmenden Überfrachtung der gemeinsprachlichen Lexik durch den immer mehr anschwellenden Fachund Bildungswortschatz nur ablenkt und wegführt, indem sie u.a. Etymologisches statt des Sprachsoziologischen hervorhebt.

Sie gründet drittens auf der Überzeugung, daß der gängige Fremdwortbegriff und das vorherrschende Fremdwortverständnis, und die damit einhergehende, wertende Polarisierung des deutschen Wortschatzes in Fremdwörter und deutsche Wörter, ihre sprachgeschichtlichen Wurzeln im 19. Jahrhundert haben, und daf diese Wurzeln mit den Wurzeln eines fremdenfeindlichen/xenophobischen Nationalgefühls verwachsen sind. Dieses Nationalgefühl blieb bis weit in das 20. Jahrhundert hinein verhängnisvoll, hat sich aber inzwischen überlebt. Das Podiumsgespräch bot eine - unerwartet aktuelle - Gelegenheit dar, erneut die Frage zu stellen, warum sich Sprachgermanisten dennoch heute immer noch einer Terminologie und Begrifflichkeit bedienen, die m.E. als na- 
tionalpolitisches Relikt gelten kann und muß. Die Thesen stellten mit anderen Worten die Frage nach der nationalpolitischen Bedeutung der Germanistik (im 19. Jahrhundert) auf der lexikalischen Ebene. Sie beschränkten sich bewuBt auf einen einzigen, wenn auch ganz zentralen, lexikalischen Punkt in dem Versuch, hier konkrete, gegenwartsbezogene Fortschritte zu erzielen. Der Versuch mißlang: In der Diskussion wurde die Frage nicht einmal angesprochen, geschweige denn einer Antwort näher geführt.

\section{Thesen}

1.1. Mit Blick auf den fremdsprachlichen lexikalischen Einfluß, teilen deutsche Sprachteilhaber heute den Wortschatz meist dichotomisch in deutsche Wörter und Fremdwörter ein. Nach herkömmlicher Auffassung sind Fremdwörter aus anderen Sprachen übernommene Wörter, ${ }^{1}$ während deutsche Wörter letztlich germanischstämmig sind. Die Dichotomie beruht somit auf dem Merkmal Herkunft und ist etymologisch begründet. Sie drückt eine Wertung aus, und berücksichtigt nur Ausdrucksseitiges. Ihre Wurzeln liegen im frühen 19. Jh., als "deutsch" auch in der Wissenschaft vielfach synonym mit "germanisch" verwendet wird, als Begriff und Bezeichnung des Fremdworts in Puristenkreisen geprägt werden.

1.2. In der Germanistik als der Wissenschaft des Deutschen (und/oder des Germanischen?) wird infolge der Erforschung vor allem des Alt(hoch)deutschen diese Einteilung meist weiter differenziert durch die Unterscheidung $z$ wischen Fremdwort und (ausdrucksseitig assimiliertem) Lehnwort, die gelegentliche Verwendung von "Erbwort" für „deutsches Wort”, und die Berücksichtigung des inneren Lehnguts bzw. der Lehnprägung. Grundmuster bleibt jedoch die Polarisierung in deutsche Wörter und Fremdwörter: Aktuelles Beispiel ist die Orthographiereformdiskussion - Schreibung der

1 Repräsentativ für die Bedeutungsangabe von Fremdwort in einem heutigen allgemeinen einsprachigen deutschen Wörterbuch ist die Angabe in der ersten Auflage des Duden 1983, S. 435: ,aus einer fremden Sprache übernom. menes (in Aussprache, Schreibweise od. Flexion noch nicht voll der überneh. menden Sprache angeglichenes) Wort". Bemerkenswert ist die abgeänderte Angabe in der 2., völlig neu bearbeiteten und stark erweiterten Auflage 1989, S. 537: "aus einer fremden Sprache übernommenes od. in der übernehmenden Sprache mit Wörtern od. Wortteilen aus einer fremden Sprache gebildetes (in Aussprache, Schreibweise od. Flexion noch nicht voll der übernehmenden Sprache angeglichenes) Wort". Der Beispielsatz ist dagegen in beiden Auflagen gleich: „übertriebener Gebrauch von Fremdwörtern". Auf einen Kommentar muß aus Raumgründen verzichtet werden. 
2.1. Kennzeichnend für das 19. Jh. (und weiterhin bis etwa Mitte des 20. Jhs.) ist eine anhaltende öffentliche Diskussion über das Fremdwort, die u.a. in zahlreichen puristischen Schriften verschiedenster Art ihren Niederschlag findet. ${ }^{3}$ Sind die Argumente gegen die Fremdwörter anfangs noch relativ sprachbezogen und differenziert, verflachen sie zusehends und zunehmend: es überwiegen nationale, bald schon nationalistische, und Fremdwörter werden im Grunde wegen ihrer (fremden) Herkunft abgelehnt, insbes. aber als Symptome und Signale unerwünschten, verderblichen fremden EinGusses auf das Deutsche und vor allem auf die Deutschen (Schweizer? Österreicher?). Sprachreinigung bzw. Fremdwortpurismus wird zum völkischen Purismus. Seltene Ausnahmen wie die Erklärung gegen den Sprachverein 1889 bestätigen nur die Regel, daß die Puristen eindeutig das Feld behaupten.

2.2. Charakteristisch für die Germanistik in diesem Zeitraum ist Ambivalenz gegenüber den Puristen, wobei im späten 19. Jh. viele Hochschullehrer sich der puristischen Bewegung anschließen oder mit ihr sympathisieren, und Abstinenz hinsichtlich (der Erforschung) des Fremdworts, und erst recht des Lehnguts im Neuhochdeutschen, was wohl u.a. mit der relativen Hervorhebung der älteren Sprachstufen und der Dialekte im Gegensatz zur nhd. Standardsprache zusammenhängt. Wiederum bestätigen seltene Ausnahmen wie Hermann Paul die allgemeine Regel. ${ }^{4}$

2 In dem Band zur Neuregelung der deutschen Rechtschreibung. Bd. 2. Hrsg. von der Kommission für Rechtschreibfragen des Instituts für deutsche Sprache wird durchgehend $z$ wischen den Bereichen ${ }_{n}$ Schreibung der Wörter (Laut-Buchstaben-Beziehung)" und "Fremdwortschreibung" unterschieden, vgl. z.B. S. 47-68 und S. 69-86 oder S. 125-145 und S. 147-169. Vgl. auch die Titelei des Rechtschreibduden: Duden Rechtschreibung der deutschen Sprache und der Fremdwörter. Diese wenigen Beispiele sind symptomatisch; es ließen sich beliebig viele andere aus allen Bereichen der Sprachbeschreibung anführen.

3 Zur Geschichte des Sprachpurismus vgl., jeweils mit weiterführender Literatur: von Polenz 1967, Kirkness 1975, Ülkü 1975, Bernsmeier 1977, Bernsmeier 1980, Bernsmeier 1983, Kirkness 1984, Schiewe 1988; vgl. auch Reichmann 1978.

4 Gemeint ist das 22. Kapitel: „Sprachmischung” seiner Prinzipien der Sprachgeschichte, S. 390-403, in dem Paul in Auseinandersetzung mit Whitney und vor allem Schuchardt die sonst übliche Fixierung auf das Fremdwort überwindet und differenziert auf verschiedene Formen des Sprachkontakts und dessen lexikalische Folgeerscheinungen eingeht. 
2.3. Kennzeichnend speziell für die Lexikographie im gleichen Zeitraum ist der auffallige Gegensatz zwischen der Vielfalt der praktischen fremdworterklärenden und vor allem -ersetzenden Gebrauchswörterbücher, wobei bezeichnenderweise "Verdeutschungswörterbuch" als Titel älter ist als „Fremdwörterbuch", und der grundsätzlichen Nichtaufnahme der Fremdwörter in eher wissenschaftlichen allgemeinen einsprachigen Wörterbüchern, wobei seltene Ausnahmen wie der germanistische Außenseiter Sanders wiederum die Regel von Adelung und Campe über Grimm u.a. - das germanistische Nationalwerk - bis zu Kluge und Trübner bestätigen. ${ }^{5}$

3. Kurzum: „Im Streit um Meidung oder Ersatz von Fremdwörtern blieben diese selbst wissenschaftlich unerforscht. Ja man kann die Behauptung wagen: durch solche Isolierung wurden sie dem natürlichen Prozeß der Integration (z.B. im Vergleich zum Englischen, Schwedischen, Niederländischen) entzogen und blieben, wofür man sie schalt: fremde Wörter" (Horst Munske ${ }^{6}$ ). Hier liegen die Wurzeln der immer noch aktuellen Fremdwortfrage und der scheinbaren Germanistenscheu vor dieser Frage. ${ }^{7}$ Von den offenkundigen Defiziten der historischen Germanistik lassen sich Desiderate der Gegenwarts- und Zukunftsgermanistik herleiten. Sie betreffen Begrifflichkeit und Terminologie, Erfassung und Beschreibung des nhd. Lehndeutsch als etymologisch begründeter Kategorie.

4.1. Zur etymologischen Erfassung des nhd. Lehndeutsch, speziell im 19. Jh., sind mindestens vier Lehngutkategorien erforderlich:

1) Wortentlehnungen und 2) Lehnprägungen einerseits, die aus zwischensprachlicher Transferenz (von Ausdruck und Inhalt (1) bzw. vom Inhalt allein (2)) aus einer Fremdsprache in das Deutsche

5 Vgl. hierzu Kirkness 1983, Kirkness 1988; speziell zum Deutschen Wörterbuch von Jacob Grimm und Wilhelm Grimm vgl. Fratzke 1987.

6 Munske 1981, S. 358.

7 Vgl. z.B. Braun 1979, S. 7: „Auf der einen Seite registriert man eine permanente öffentliche Beschäftigung mit schier unlösbaren Fremdwortfragen; daraus kann man - zumindest indirekt - auf real bestehende Informationsbedürfnisse schließen. Auf der anderen Seite beobachtet man den bedauerlichen Tatbestand, daß wissenschaftliche Disziplinen wie Sprachwissenschaft und Germanistik sich fast gar nicht mit dem Themenkomplex beschäftigen. Die Fremdwort-Diskussion ist an vielen Hochschulen kein Thema; sie findet keinen Platz im Fächerkatalog der Disziplinen und kaum Zugang zu Hochschulseminaren. Daher fehlt es bis heute an sprachwissenschaftlich gesicherten Gesamtdarstellungen zu einem Themenbereich mit einer besonders ausgeprägten Interessenstruktur in der Öffentlichkeit." 
resultieren; sowie 3) Lehnwortbildungen und 4) Lehnersatzwörter andererseits, die sich aus innereinzelsprachlichen Entwicklungen deutschen Lehnguts (vor allem Wortentlehnungen) nach der Übernahme ins Deutsche ergeben.

4.2. Die Wurzeln des heutigen Lehndeutsch liegen deutlich vor dem 19. Jh., wobei die entscheidende Schwelle zur Gegenwart um $1770 \mathrm{zu}$ liegen scheint. Wortentlehnungen und Lehnprägungen sind von ahd. Zeit bis in die Gegenwart zwar in unterschiedlicher Stärke, dennoch konstant belegt. Lehnwortbildungen sind vor allem in nhd. Zeit zunehmend nachweisbar. (In puristischer Absicht geprägte) Lehnersatzwörter dagegen sind Spezifika des Nhd. von ca. 1600-1940. $\mathrm{Zu}$ den Wortentlehnungen und Lehnwortbildungen (= Fremdwörtern) im Nhd., bes. im 19. Jh., vgl. die nachfolgenden Tabellen aus dem DFWB (3.).

4.3. Wissenschaftlich erforscht ist das deutsche Lehngut des 19. Jhs. (und weiterhin bis etwa Mitte des 20. Jhs.) trotz der umfangreichen Fremdwortliteratur und -lexikographie nur in Ansätzen. Relativ gut dokumentiert sind lediglich die Lehnersatzwörter, ${ }^{8}$ sehr schlecht dagegen die Lehnprägungen. ${ }^{9}$ Hinsichtlich der Wortentlehnungen und Lehnwortbildungen ist der Germanist vorerst auf einige wenige (Fremd-)Wörterbücher angewiesen, allen voran das DFWB 1913-88, im 19. Jh. daneben Sanders 1871 (und 1860-65) und Kehrein 1876. ${ }^{10}$ Auf Primärquellen beruhende, wissenschaftlichen Ansprüchen genügende Spezialstudien zu Entlehnungen aus den einflußreichsten einzelnen Herkunftssprachen, vor allem Englisch und Französisch, gibt es bis heute ebensowenig wie theoretisch und empirisch abgesicherte historisch-diachrone Untersuchungen zu den Lehnwortbildungen. ${ }^{11}$

8 Vgl. z.B. (die Wortlisten in) Steuernagel 1926, Holz 1950, Kamb-Spies 1962, Kirkness 1975.

- Für Lehnwörter und Lehnprägungen aus dem Russischen vgl. Kohls 1964; hinsichtlich des 19. (und des 20.) Jhs. handelt die Arbeit von Kamb-Spies 1962 von den puristischen Verdeutschungen, also den Lehnersatzwörtern im Gegensatz zu den Lehnprägungen.

10 Sanders 1860-1865, Sanders 1871, Kehrein 1876; vgl. hierzu Kirkness 1988, S. 715-718.

11 Im Institut für deutsche Sprache wird zur Zeit ein Lexikon der LehnWortbildung erarbeitet, das hinsichtlich der Lehnwortbildungen Abhilfe schaffen wird. Vgl. hierzu Hoppe/Kirkness/Link/Nortmeyer/Rettig/ Schmidt 1987, Link 1988. 
5. Die historisch-diachrone wissenschaftliche Erforschung des Lehndeutschen, auch in seinem Verhältnis zum Indigendeutschen, setzt die Überwindung der (im nachpuristischen Zeitalter ungerechtfertigten) Fixierung auf das Fremdwort voraus, und ist selbst wiederum eine Voraussetzung für eine zeit- und sachgemäße Antwort auf die sog. Fremdwortfrage in der Synchronie. Diese tut not, denn die vom 19. Jh. herrührende national-puristische 'Antwort' ist keine. ${ }^{12}$

\section{Tabellen}

Es folgen Tabellen zum Verhältnis von Wortentlehnungen und Lehnwortbildungen im Neuhochdeutschen, bes. im 19. Jh.

Die Tabellen enthalten Annäherungswerte, keine absoluten Zahlen aus dem 'Deutschen Fremdwörterbuch' (DFWB) ${ }^{13}$.

12 Bereits 1967 hat Peter von Polenz den Weg zu einer wissenschaftlich fundierten, sach- und zeitgemäßen Fremdwortbehandlung in der Synchronie gezeigt; vgl. von Polenz 1967.

Deutsches Fremdwörterbuch 1913-1988; wegen der drei verschiedenen Bearbeitungsphasen, A-K durch Hans Schulz (1913), L-P und Q durch Otto Basler (1942, 1972) und R-Z im IDS (1977-1983) und der sich dadurch jeweils verschiebenden "Gegenwarts" sprache wird in der zweiten Tabelle zwischen den Buchstabenstrecken $A-Q$ und $R-Z$ unterschieden. 


\begin{tabular}{|c|c|c|c|}
\hline 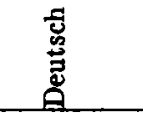 & 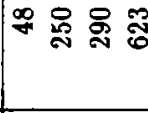 & $\vec{\Xi}$ & 옹 \\
\hline 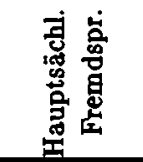 & 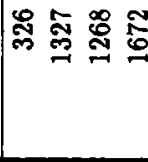 & 袩 & 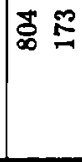 \\
\hline స્త & 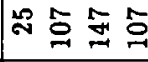 & $\stackrel{\infty}{\infty}$ & I \\
\hline 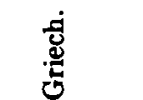 & 萬 & F & 8 \\
\hline 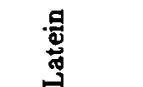 & 蛋 & 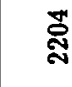 & $\stackrel{20}{2}$ \\
\hline 㟃 & $1-\approx$ & 号 & $\underset{\infty}{\approx}=$ \\
\hline รี่ & 유 욤 융 ஜ & $\underset{\text { ஸ્ }}{\infty}$ & $\underset{\infty}{\infty}$ \\
\hline / & 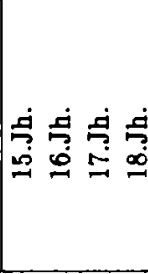 & 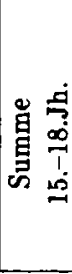 & 官 \\
\hline
\end{tabular}




\begin{tabular}{|c|c|c|c|c|c|c|}
\hline 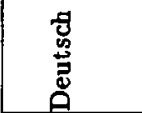 & 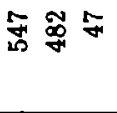 & $\stackrel{0}{0}$ & 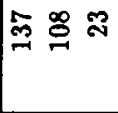 & 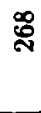 & 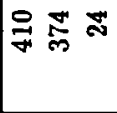 & $\stackrel{\infty}{\infty}$ \\
\hline 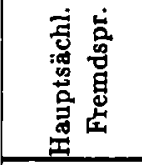 & 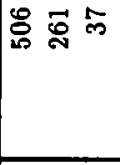 & $\overleftarrow{\infty}_{\infty}$ & 第 & $\underset{乛}{*}$ & : & ஜ্ণ \\
\hline 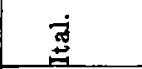 & $=\equiv-$ & g্ণ & $\Rightarrow \infty 1$ & ָิ & 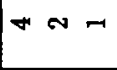 & $r$ \\
\hline 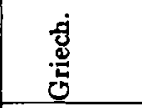 & $\hat{n} \varrho$ & 8 & 品 & $\mathscr{z}$ & $-\infty r$ & $\approx$ \\
\hline 㞼 & 头 20 & 禺 & $\stackrel{N}{*}$ & $\stackrel{8}{8}$ & $\approx \infty$ & $g$ \\
\hline 它 & $\vec{\sigma} \ddot{\infty} \infty$ & $\underset{\sim}{\infty}$ & $\approx \infty \infty$ & ช & $\mathbb{f} \infty$ & 8 \\
\hline 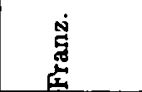 & 品号 & $\stackrel{\infty}{\infty}$ & $\infty \underset{\infty}{\infty} \cong$ & $\underset{\sim}{\infty}$ & に岕 & 5 \\
\hline 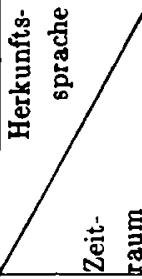 & 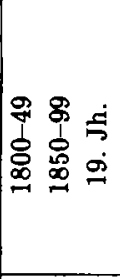 & 昌 & 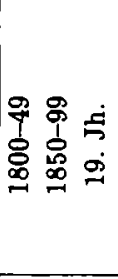 & 宸 & 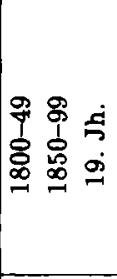 & $\begin{array}{c}\mathscr{Q} \\
\stackrel{\Xi}{\Xi} \\
\stackrel{\Xi}{\Xi}\end{array}$ \\
\hline 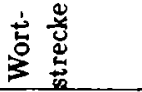 & $\frac{N}{1}$ & & $\begin{array}{l}0 \\
1 \\
4\end{array}$ & & $\begin{array}{c}N \\
1 \\
x\end{array}$ & \\
\hline
\end{tabular}




\section{Literatur}

Bernsmeier, Helmut (1977): Der Allgemeine Deutsche Sprachverein in seiner Gründungsphase. In: Muttersprache 87, S. 369-395.

Bernsmeier, Helmut (1980): Der Algemeine Deutsche Sprachverein in der Zeit von 1912 bis 1932. In: Muttersprache 90, S. 117-140.

Bernsmeier, Helmut (1983): Der Deutsche Sprachverein im „Dritten Reich”. In: Muttersprache 93, S. 31-58.

Braun, Peter (Hrsg.) (1979): Fremdwort-Diskussion. München.

Deutsches Fremdwörterbuch (1913-1988): Deutsches Fremdwörterbuch. Begründet von Hans Schulz, fortgeführt von Otto Basler, weitergeführt im Institut für deutsche Sprache. Bd. 1-7. (Straßburg) Berlin/New York.

Duden (1983, 1989): Duden. Deutsches Universalwörterbuch. 1. Aufl. 1983. 2. völlig neu bearbeitete und stark erweiterte Aufl. 1989. Mannheim/Wien/Zürich.

Fratzke, Ursula (1987): Zum Fremdwort im Deutschen Wörterbuch. In: Dückert, Joachim (Hrsg.): Das Grimmsche Wörterbuch. Untersuchungen zur lexikographischen Methodologie. Leipzig/Stuttgart, S. 153-169.

Holz, Guido (1950): Joachim Heinrich Campe als Sprachreiniger und Wortschöpfer. Diss. (masch.). Tübingen.

Hoppe, Gabriele/Kirkness, Alan/Link, Elisabeth/Nortmeyer, Isolde/Rettig, Wolfgang/Schmidt, Günter Dietrich (1987): Deutsche Lehnwortbildung. Beiträge zur Erforschung der Wortbildung mit entlehnten WB-Einheiten im Deutschen. Tübingen (Forschungsberichte des Instituts für deutвche Sprache 64).

Kamb-Spies, Renate (1962): Lehnprägungen der deutschen Sprache. Diss. (masch.). Tübingen.

Kehrein, Josef (1876): Fremdwörterbuch mit etymologischen Erklärungen und zahlreichen Belegen aus Deutschen Schriftstellern. Stuttgart.

Kirkness, Alan (1975): Zur Sprachreinigung im Deutschen 1789-1871. Eine historische Dokumentation. 2. Bde. Tübingen (Forschungsberichte des Instituts für deutsche Sprache 26, 1/2).

Kirkness, Alan (1983): Zur germanistischen Fremdwortlexikographie im 19./20. Jahrhundert: Bibliographie der Fremd- und Verdeutschungswörterbücher 1800-1945. In: Germanistische Linguistik 1-3, S. 113-174. 
Kirkness, Alan (1984): Das Phänomen des Purismus in der Geschichte des Deutschen. In: Besch, Werner/Reichmann, Oskar/Sonderegger, Stefan (Hrsg.): Sprachgeschichte. Ein Handbuch zur Geschichte der deutschen Sprache und ihrer Erforschung. Bd. 1. Berlin/New York, S. 290-299.

Kirkness, Alan (1988): Nachwort „Deutsches Fremdwörterbuch” - eine historische Dokumentation als Beitrag zur Geschichte der germanistischen (Fremdwort-)Lexikographie. In: Deutsches Fremdwörterbuch. Bd. 7, S. 701-840.

Kohls, Siegfried (1964): Russisches lexikalisches Lehngut im deutschen Wortschatz (der letzten vier Jahrhunderte). Diss. (masch.). Berlin.

Kommission für Rechtschreibfragen des Instituts für deutsche Sprache (Hrsg.) (1989): Vorschlag zur Neuregelung der deutschen Rechtschreibung. Der kommentierte Vorschlag der Kommission für Rechtschreibfragen des Instituts für deutsche Sprache, Mannheim, und die Stellungnabme der Gesellschaft für deutsche Sprache, Wiesbaden. Düsseldorf (Sprache der Gegenwart 77).

Link, Elisabeth (1988): Lehnwortbildung im Wörterbuch. In: Harras, Gisela (Hrsg.): Das Wörterbuch. Artikel und Verweisstrukturen. Jahrbuch 1987 des Instituts für deutsche Sprache. Düsseldorf (Sprache der Gegenwart 74), S. 223-264.

Munske, Horst (1981): Rezension des Deutschen Fremdwörterbuchs (Bd. 3-5). In: Zeitschrift für Dialektologie und Linguistik 48, S. 357-359.

Paul, Hermann (1966): Prinzipien der Sprachgeschichte. 7. Auf. Tübingen.

von Polenz, Peter (1967): Sprachpurismus und Nationalismus. Die 'Fremdwort'-Frage gestern und heute. In: von Wiese, Benno/Hens, Richard (Hrsg.): Nationalismus in Germanistik und Dichtung. Dokumentation des Germanistentages in München vom 17.-22. Oktober 1966. Berlin, S. 79-112.

Reichmann, Oskar (1978): Deutsche Nationalsprache. Eine kritische Darstellung. In: Germanistische Linguistik 2-5, S. 389-423.

Sanders, Daniel (1860-1865): Wörterbuch der deutschen Sprache. 3 Bde. Leipzig.

Sanders, Daniel (1871): Fremdwörterbuch. 2 Bde. Leipzig.

Schiewe, Jürgen (1988): Sprachpurismus und Emanzipation: J.H. Campes Verdeutschungsprogramm. Hildesheim/Zürich/New York (Germanistische Linguistik 96-97). 
Steuernagel, Otto (1926): Die Einwirkungen des Deutschen Sprachvereins auf die deutsche Sprache. Berlin (Wissenschaftliche Beihefte zur Zeitschrift des Deutschen Sprachvereins 41).

Ülkü, Vural (1975): Sprachreinigungsbestrebungen in Deutschland seit den 80 er Jahren des 19. Jahrhunderts bis zur Gegenwart. Ankara. 